\title{
A New Semantics for Systems of Logic of Essence
}

\author{
Alessandro Giordani \\ Università Cattolica di Milano
}

\begin{abstract}
.
The purpose of the present paper is to provide a way of understanding systems of logic of essence by introducing a new semantic framework for them. Three central results are achieved: first, the now standard Fitting semantics for the propositional logic of evidence is adapted in order to provide a new, simplified semantics for the propositional logic of essence; secondly, we show how it is possible to construe the concept of necessary truth explicitly by using the concept of essential truth; finally, Fitting semantics is adapted in order to present a simplified semantics for the quantified logic of essence.
\end{abstract}

Keywords: essence; logic of essence; necessity; possible worlds semantics; Fitting semantics.

\section{Introduction}

It is always interesting when logical systems developed in different areas of research and for different purposes are discovered to be connected in some significant respect. In this paper we show how systems of logic of essence, both propositional and quantified, can be provided with a specific kind of semantics which was introduced in order to interpret systems of logic of evidence (see [1] and [7] for extended presentations). As a consequence, the logic of essence turns out to be a sort of counterpart to the logic of evidence, where the problem of closure under necessary implications plays the role of the problem of closure under analytical implications.

\subsection{The logic of essence}

The logic of essence was introduced by Fine ([5], [6]; see also [3], [4] to understand the philosophical background) in order to capture the distinction between objects having a property and objects having a property in virtue of their essence. This distinction, according to Fine, cannot be construed as a difference between objects having a property and objects having a property by necessity. To be sure, while it is accepted that, if an object essentially has a certain property, then it has that property by necessity, the converse proposition is found to be problematic, primarily because the concept of necessity, in contrast to the concept of essence, is insensitive to variations in source (see, in particular, [3]). Thus, it makes sense to say that Socrates is, by necessity, the unique member of the singleton set containing him, whereas it seems to offend our intuitions about what is essential to Socrates to say that Socrates is, in virtue of his essence, the unique member of the singleton set. Actually, the source of the necessity of that membership is to be traced back to the essence of the singleton set containing Socrates, not to the essence of Socrates himself. This conclusion can be generalized by stating that the necessity of the truth of a proposition is rooted in the essence of the entities which the proposition is about. 


\subsection{The concept of essential truth}

In [5] a proposition of the form $[X] \varphi$ is interpreted as saying that $\varphi$ is true in virtue of the essence of some entities in $X$. The truth conditions of $[X] \varphi$ can be stated by exploiting a reflexive and transitive relation of ontological dependence (see [6] section 3) and saying, roughly, that $[X] \varphi$ is true, i.e. $\varphi$ is true in virtue of the essence of some entities in $X$, if and only if $\varphi$ is true in every world in which the entities in $X$ exist and each constituent of $\varphi$ is such that some element of $X$ depends on it ${ }^{1}$. Yet, as suggested by Fine, instead of defining the concept of essential truth by using the ontological dependence relation, we can introduce the concept as a primitive concept. If we follow this strategy, three key problems arise:

(1) the problem of characterizing the concept of essence;

(2) the problem of defining the concept of ontological necessity in terms of essential truth; and

(3) the problem of defining the concept of ontological dependence in terms of essential truth.

As we will see, a development of Fitting semantics will allow us both to provide an elegant and flexible semantics for systems of logic of essence and to deal with the previous problems.

\subsection{A new semantic framework for the concept of essential truth}

Within the standard semantic framework of modal logic the following characterization of the notion of necessary truth is expanded:

$p$ is necessarily true $\quad \approx \quad p$ is true in every metaphysically possible world

In a similar way, in the standard semantic framework of epistemic logic, the following characterization of the notion of knowledge is considered:

$$
p \text { is known to be true } \quad \approx \quad p \text { is true in every epistemically possible world }
$$

If we adopt the view that the source of knowledge is evidence, we are led to assume the following analysis of knowledge ${ }^{2}$ :

$$
p \text { is known to be true } \approx p \text { is accepted as true on the basis of some justifier }
$$

In a similar way, if we adopt the view that the source of necessity is the essence of a certain entity, we are led to assume the following analysis of necessary truth:

\footnotetext{
${ }^{1}$ See [3] for an extended introduction. Here the condition that $\varphi$ is necessarily true is added in order to determine which proposition is true, since, as we will see, both $\varphi$ and its negation have the same constituents. This condition is consistent with the view proposed by Fine in [3], according to which if an object essentially has a certain property then it is necessary that it has that property.

${ }^{2}$ See [1].
} 
$p$ is necessarily true $\quad \approx \quad p$ is true on the basis of the essence of some entity

Now, since the problems and intuitions that give rise to the axiomatic analysis of the property of being true on the basis of essence are similar to the ones that give rise to the axiomatic analysis of the property of being accepted on the basis of a justifier, we are encouraged to develop the logic of essence along the lines of the logic of evidence. In particular, we can assume that every set of basic entities is characterized by the set of propositions that are true in virtue of the identity of those entities, and that the set of necessary truths can be defined in terms of the union of the sets of propositions associated with sets of basic entities that exist in a certain world.

The rest of this paper is aimed at developing these assumptions and is organized as follows. In section 2 a system $\mathbf{E}$ of propositional logic of essence is introduced and proved to be sound and strongly complete with respect to an appropriate semantics which is developed along the lines of Fitting semantics for the logic of evidence. In addition, it is shown that $\mathbf{E}$ is equivalent to a system ED of propositional logic of essence which includes the notion of ontological dependence, and that the concept of necessary truth can be interpreted on the basis of the concept of essential truth. In section $3 \mathbf{E}$ is extended to a system $\mathbf{E}^{=}$of propositional logic of essence that essentially coincides with the system proposed in [2], which is then interpreted according to the proposed semantics. In section 4 we show how these systems of propositional logic of essence can be developed to systems of quantified logic of essence, which are then proved to be sound and strongly complete with respect to an extension of the semantics of $\mathbf{E}$ and $\mathbf{E}^{=}$. Finally, in section 5 the advantages and disadvantages of the proposed semantics are briefly discussed, and future lines of research are proposed.

\section{The basic systems}

In this section we will introduce a basic system $\mathbf{E}$ of propositional logic of essence. The alphabet of the language $L(\mathbf{E})$ of $\mathbf{E}$ contains the following signs:

- a countable set $P V$ of variables for propositions $\left(p, p^{\prime}, p^{\prime \prime}, \ldots\right)$.

- a countable set $D V$ of variables for delimiters $\left(d, d^{\prime}, d^{\prime \prime}, \ldots\right)$.

- a constant for the universal delimiter: $V$.

- the composition operator: $\bullet$.

- the constitution operator: ||.

- the predicate of existence: $\boldsymbol{E x}$.

- the modality constructor: [ ].

- brackets: ( and ).

The sets $\operatorname{Tm}(\mathbf{E})$ and $F m(\mathbf{E})$ of terms and formulas of $L(\mathbf{E})$ are defined by simultaneous induction according to the following formation rules. 
Rules for terms:

$X:=d|V| X \cdot Y|| \varphi \mid$

where $d \in D V$ and $\varphi \in F m(\mathbf{E})$

Rules for formulas:

$\varphi:=p|\neg \varphi|(\varphi \wedge \varphi)|\boldsymbol{E x}(X)|[X] \varphi \quad$ where $p \in P V$ and $X \in \operatorname{Tm}(\mathbf{E})$

The other propositional connectives are introduced in the usual way.

On the intended interpretation, a basic delimiter stands for a plurality ${ }^{3}$ of basic entities, $V$ is the plurality of all basic entities and $X \bullet Y$ is the plurality of the entities that are in $X$ and $Y$. Finally, $|\varphi|$ is the plurality of the constituents of proposition $\varphi$, or the plurality of all basic entities that constitute the situation connected to $\varphi$. There is no assumption concerning the identity and ontological status of the basic entities. In particular, we are not assuming that the basic entities are individuals and that delimiters are to be intended as a plurality of individuals. Finally, $\boldsymbol{E} \boldsymbol{x}(X)$ states that $X$ is a possibly existent plurality of basic entities, where the predicate of existence is assumed to refer to the possible existence of an entity in a certain world (see [6], p. 543). ${ }^{4}$

\subsection{Axioms}

System $\mathbf{E}$ is determined by the following groups of axioms and rules ${ }^{5}$.

Group I: classical axioms and modus ponens.

Group II: modal axioms and rules.

\begin{tabular}{|l|l|}
\hline Axioms on delimiters & Axioms on modalities \\
\hline D1: $[X] \varphi \leftrightarrow[X \bullet X] \varphi$. & M1: $[X]\left(\varphi \rightarrow \varphi^{\prime}\right) \wedge[X] \varphi \rightarrow[X] \varphi^{\prime}$. \\
D2: $[X \bullet Y] \varphi \leftrightarrow[Y \bullet X] \varphi$. & M2: $[X] \varphi \rightarrow[X \bullet Y] \varphi$. \\
D3: $\left[X \bullet\left(Y \bullet Y^{\prime}\right)\right] \varphi \leftrightarrow\left[(X \bullet Y) \bullet Y^{\prime}\right] \varphi$. & M3: $[X] \varphi \rightarrow \varphi$. \\
D4: $[X \bullet V] \varphi \leftrightarrow[V] \varphi$. & M4: $[X] \varphi \rightarrow[X][X] \varphi$. \\
D5: $[|\neg \varphi|] \varphi^{\prime} \leftrightarrow[|\varphi|] \varphi^{\prime}$. & M5: $[X \bullet|\varphi|][X] \varphi \vee[X \bullet|\varphi|] \neg[X] \varphi$. \\
D6: $\left[\left|\varphi \wedge \varphi^{\prime}\right|\right] \varphi^{\prime \prime} \leftrightarrow\left[|\varphi \cdot| \varphi^{\prime} \mid\right] \varphi^{\prime \prime}$. & M6: $[X] \varphi \wedge[Y \bullet|\varphi|] \varphi^{\prime} \rightarrow[Y \bullet X] \varphi^{\prime}$. \\
D7: $[|\boldsymbol{E x}(X)|] \varphi \leftrightarrow[X] \varphi$. & ME: $\boldsymbol{E x}(X)$. \\
D8: $[|[X] \varphi|] \varphi^{\prime} \leftrightarrow[X \bullet|\varphi|] \varphi^{\prime}$. & RM: $|-\varphi=>|-[|\varphi|] \varphi$. \\
\hline
\end{tabular}

\footnotetext{
${ }^{3}$ Intuitively, a plurality is just a collection of entities and it is to be interpreted as a collection as many (and contrasted with a collection as one, i.e. a sum). Alternatively, one can intend delimiters as names for classes (see [2], §I).

${ }^{4}$ If we had quantifiers for delimiters and the identity relation, $\boldsymbol{E} \boldsymbol{x}(X)$ could be defined as $\exists Y(X=Y)$. This definition justifies axiom ME, which states that every plurality exists.

5 The following axioms, with the exception of ME, are either included or derivable both in the system of propositional logic of essence proposed in [2] and in the system of quantified logic of essence proposed in [5].
} 
Axioms on delimiters characterize the composition operator and the constitution operator. According to the intended interpretation, the composition operator takes pluralities of basic entities and returns their composition. It is then expected that composition is idempotent, commutative and associative, and that $V$ is a top element. As to the constitution operator, we make the intuitive assumptions that the constituents of a composed proposition coincide with the plurality of the constituents of the component propositions. Axioms on modalities provide a basic characterization of essential truth. M1 states that, if both an implication $\varphi \rightarrow \varphi^{\prime}$ and its antecedent are true in virtue of the essence of some entities in $X$, then the consequent $\varphi$ ' is also true in virtue of the essence of some entities in $X$. M2 states that what is true in virtue of the essence of some entities in $X$ is true in virtue of the essence of some entities in any plurality of entities that includes $X$. M3 states that what is true in virtue of the essence of some entities is true, while M4 states that the truth of a modal proposition $[X] \varphi$ is determined by entities in $X$ and $\mathbf{M 5}$ states that the truth value of $[X] \varphi$ is completely determined by the entities in $X \bullet|\varphi|$. M6 (the cut principle proposed in [5]) is a generalization of the crucial principle according to which, if a proposition $\varphi$ is true in virtue of the essence of some entities in $X$ and another proposition $\varphi$ ' is true in virtue of the essence of some constituents of $\varphi$, then $\varphi^{\prime}$ is also true in virtue of the essence of some entities in $X$. Finally, ME states that every plurality exists and RM states that any logical truth is true in virtue of some of its own constituents.

\subsection{Theorems}

Some useful theorems of $\mathbf{E}$ are the following ones.

T1: $\mid-[X] \varphi \rightarrow[V] \varphi$.

$-[X] \varphi \rightarrow[X \cdot V] \varphi$, by M2.

$-[X] \varphi \rightarrow[V] \varphi$, by $\mathbf{D} 4$.

T2: $\mid-[X] \varphi \rightarrow[V][X] \varphi$.

$\mid-[X] \varphi \rightarrow \neg[X \bullet|\varphi|] \neg[X] \varphi$, by M3.

$-[X] \varphi \rightarrow[X \bullet|\varphi|][X] \varphi$, by M5.

$-[X] \varphi \rightarrow[V][X] \varphi$, by $\mathbf{T 1}$.

T3: $\mid-\neg[X] \varphi \rightarrow[V] \neg[X] \varphi$.

$\mid-[X] \varphi \rightarrow \neg[X \bullet|\varphi|][X] \varphi$, by M3.

$\mid-\neg[X] \varphi \rightarrow[X \bullet|\varphi|] \neg[X] \varphi$, by M5.

$\mid-\neg[X] \varphi \rightarrow[V] \neg[X] \varphi$, by T1.

T4: $\mid-[X]\left(\varphi \rightarrow \varphi^{\prime}\right) \wedge[Y] \varphi \rightarrow[X \cdot Y] \varphi^{\prime}$.

$\mid-[X \bullet Y]\left(\varphi \rightarrow \varphi^{\prime}\right) \wedge[X \bullet Y] \varphi \rightarrow[X \bullet Y] \varphi^{\prime}$, by M1.

$-[X \bullet Y]\left(\varphi \rightarrow \varphi^{\prime}\right) \wedge[Y] \varphi \rightarrow[X \bullet Y] \varphi^{\prime}$, by M2.

$-[X]\left(\varphi \rightarrow \varphi^{\prime}\right) \wedge[Y] \varphi \rightarrow[X \bullet Y] \varphi^{\prime}$, by M2. 
T5: $\mid-[X] \varphi \wedge[|\varphi|] \varphi^{\prime} \rightarrow[X] \varphi^{\prime}$,

$\mid-[X] \varphi \wedge\left[X^{\bullet}|\varphi|\right] \varphi^{\prime} \rightarrow[X \bullet X] \varphi^{\prime}$, by M6.

$\mid-[X][X] \varphi \wedge\left[X^{\bullet}|\varphi|\right] \varphi^{\prime} \rightarrow[X] \varphi^{\prime}$, by D1.

$\mid-[X] \varphi \wedge[|\varphi|] \varphi^{\prime} \rightarrow[X] \varphi^{\prime}$, by M2.

T6: $\mid-[Y][X] \varphi \rightarrow[Y] \varphi$.

$\mid-[|[X] \varphi \rightarrow \varphi|]([X] \varphi \rightarrow \varphi)$, by M3 and RM.

$\mid-[|[X] \varphi|]([X] \varphi \rightarrow \varphi)$, by D6 and D8.

$-[Y][X] \varphi \rightarrow[|[X] \varphi| \cdot Y] \varphi$, by T5.

$-[Y][X] \varphi \rightarrow[Y \bullet Y] \varphi$, by M5.

$-[Y][X] \varphi \rightarrow[Y] \varphi$, by $\mathbf{D 1}$.

T7: [ $V]$ is an $S 5$ modality.

$|-\varphi=>|-[V] \varphi$, by $\mathbf{R M}$ and $\mathbf{M} \mathbf{2}$.

$-[V]\left(\varphi \rightarrow \varphi^{\prime}\right) \wedge[V] \varphi \rightarrow[V] \varphi^{\prime}$, by $\mathbf{M 1}$.

$-[V] \varphi \rightarrow \varphi$, by $\mathbf{M 3}$.

$-[V] \varphi \rightarrow[V][V] \varphi$, by $\mathbf{T} 2$.

$\mid-\neg[V] \varphi \rightarrow[V] \neg[V] \varphi$, by $\mathbf{T 3}$.

T8: $\varphi_{1}\left|-\varphi=>\left[X_{1}\right] \varphi_{1}\right|-\left[|\varphi| \cdot X_{1}\right] \varphi$.

$-\varphi_{1} \rightarrow \varphi$, by hypothesis.

$\mid-\left[\left|\varphi_{1} \rightarrow \varphi\right|\right]\left(\varphi_{1} \rightarrow \varphi\right)$ by RM.

$\mid-\left[\left|\varphi_{1}\right| \cdot|\varphi|\right]\left(\varphi_{1} \rightarrow \varphi\right)$ by D6 and D8.

$-\left[X_{1}\right] \varphi_{1} \rightarrow\left[\left|\varphi_{1}\right| \bullet|\varphi| \cdot X_{1}\right] \varphi$, by T5.

$-\left[X_{1}\right] \varphi_{1} \rightarrow\left[|\varphi| \cdot X_{1}\right] \varphi$, by M6.

Corollary: $\varphi_{1}, \ldots, \varphi_{\mathrm{n}}\left|-\varphi=>\left[X_{1}\right] \varphi_{1}, \ldots,\left[X_{\mathrm{n}}\right] \varphi_{\mathrm{n}}\right|-\left[|\varphi| \cdot X_{1} \bullet \ldots \bullet X_{\mathrm{n}}\right] \varphi$

\subsection{Semantics}

The semantics of the logic of essence is an adaptation of Fitting semantics for the logic of evidence. In particular, a frame for the logic of essence is a Fitting frame combined with a constituency $\operatorname{model}^{6}$ that allows us to interpret the terms in $\operatorname{Tm}(\mathbf{E})$.

Definition 2.3.1: constituency structure.

A constituency structure $\boldsymbol{B}=(B,+, 1)$ is a joint semilattice with one.

Hence, + is a binary operation on $B$ satisfying

$+1: b+b=b$

$+2: b+b^{\prime}=b^{\prime}+b$

\footnotetext{
${ }^{6}$ The notion of constituency model is introduced in [2].
} 
$+3: b+\left(b^{\prime}+b^{\prime \prime}\right)=\left(b+b^{\prime}\right)+b^{\prime \prime}$

$+4: b+1=1$

As usual, a partial order $\leq$ on $B$ can be defined by $b \leq b^{\prime}<=>b+b^{\prime}=b^{\prime}$. The elements of $B$ can be identified with pluralities of basic entities. Hence, the operation + represents the composition of pluralities and the top element represents the plurality of all basic entities.

Definition 2.3.2: constituency model.

A constituency model is a pair $\mathrm{B}=(\boldsymbol{B}, \mathfrak{\Im})$, where:

i) $\boldsymbol{B}$ is a constituency structure

ii) $\mathfrak{\Im}$ is a function from terms to elements of $B$ which satisfies:

$\mathfrak{I} 1: \mathfrak{I}(V)=1$

$\mathfrak{I} 2: \mathfrak{I}(X \cdot Y)=\mathfrak{I}(X)+\mathfrak{I}(Y)$

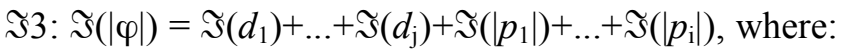

a) $d_{1}, \ldots, d_{\mathrm{j}}$ are the variables for delimiters occurring in $\varphi$.

b) $p_{1}, \ldots, p_{\mathrm{i}}$ are the variables for propositions occurring in $\varphi$.

Definition 2.3.3: frame for $L(\mathbf{E})$.

A frame for $L(\mathbf{E})$ is a tuple $(W, R, \mathrm{~B}, E)$, where:

i) $W$ is the set of possible worlds.

ii) $R \subseteq W \times W$ is an equivalence relation on $W$.

iii) $\mathrm{B}=(B,+, 1, \mathfrak{I})$ is a constituency model.

iv) $E: W \times B \rightarrow \wp(F m(\mathbf{E}))$ is a selection function respecting the following conditions:

$E 1: \varphi \rightarrow \varphi^{\prime} \in E(w, b)$ and $\varphi \in E(w, b)=>\varphi^{\prime} \in E(w, b)$.

$E 2: E(w, b) \subseteq E\left(w, b+b^{\prime}\right)$.

$E 3: E(w, 1)$ is consistent.

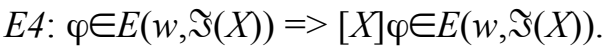

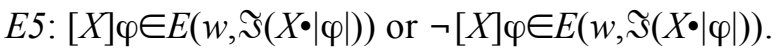

$E 6: \varphi \in E(w, b)=>E\left(w, b^{\prime}+\Im(|\varphi|)\right) \subseteq E\left(w, b^{\prime}+b\right)$.

$E R$ (Reflexivity): $[X] \varphi \in E(w, b)=>\varphi \in E(w, b)$.

$E S$ (Stability): $R(w, v)=>E(w, b)=E(v, b)$.

Intuitively, two worlds are connected by $R$ when they are indistinguishable with respect to the propositions whose truth value is fixed by all the basic entities. In addition, the set of formulas assigned by $E$ to entities in a certain world represents the set of propositions whose truth is determined in virtue of the essence of such entities. 
Definition 2.3.4: model for $L(\mathbf{E})$.

A model for $L(\mathbf{E})$ is a tuple $M=(W, R, \mathrm{~B}, E, V)$, where $V: P V \rightarrow \wp(W)$ is a modal valuation.

Definition 2.3.5: truth (in a model at a world).

$M, w \mid=p<=>w \in V(p)$.

$M, w|=\neg \varphi<=>\operatorname{not} M, w|=\varphi$.

$M, w\left|=\varphi \wedge \varphi^{\prime}<=>M, w\right|=\varphi$ and $M, w \mid=\varphi^{\prime}$.

$M, w \mid=\boldsymbol{E} \boldsymbol{x}(X)<=>\Im(X) \in B$, i.e. $M, w \mid=\boldsymbol{E} \boldsymbol{x}(X)$ for all $X$.

$M, w \mid=[X] \varphi<=\forall v \in W(R(w, v)=>M, v \mid=\varphi)$ and $\varphi \in E(w, \Im(X))$.

A formula $\varphi$ is said to be true in a model, $M \mid=\varphi$, when it is true at every world in the model, and it is said to be valid in a class of models when it is true in every model of that class.

Theorem 2.3.1: $M, w \mid=[X] \varphi<\Rightarrow \forall v \in W(R(w, v)=>M, v \mid=[X] \varphi)$.

Suppose $M, w \mid=[X] \varphi$, i.e. $\forall x \in W(R(w, x)=>M, x \mid=\varphi)$ and $\varphi \in E(w, \Im(X))$.

$R$ is an equivalence relation, by definition 2.3.3.

Thus $R(w, v)=>\forall x \in W(R(v, x) \Rightarrow M, x \mid=\varphi)$.

$R(w, v)=>E(w, \Im(X))=E(v, \Im(X))$, by stability.

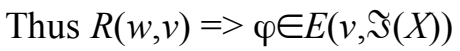

Therefore, $R(w, v)=>M, v \mid=[X] \varphi$.

The other direction follows from the reflexivity of $R$.

Theorem 2.3.2: $M, w \mid=\neg[X] \varphi<\Rightarrow \forall v \in W(R(w, v)=>M, v \mid=\neg[X] \varphi)$.

Suppose not $M, w \mid=[X] \varphi$, i.e. $\exists x \in W(R(w, x)$ and not $M, x \mid=\varphi)$ or $\varphi \notin E(w, \Im)(X))$.

$R$ is an equivalence relation, by definition 2.3.3.

Thus $R(w, v)=>\exists x \in W(R(v, x)$ and not $M, x \mid=\varphi)$

$R(w, v)=>E(w, \Im(X))=E(v, \Im(X))$, by stability.

Thus $R(w, v)=>\varphi \notin E(w, \Im(X))$.

Therefore, $R(w, v)<=>\operatorname{not} M, v \mid=[X] \varphi$.

The other direction follows from the reflexivity of $R$.

Definition 2.3.6: universality condition (UC).

$M$ satisfies $U C \Leftrightarrow \forall \forall(M \mid=\varphi \Rightarrow \forall w \in W(\varphi \in E(w, \mathfrak{\Im}(|\varphi|)))$.

$U C$ states that a proposition that is true at all the worlds of a model is true in virtue of the essence of its constituents at each world.

Definition 2.3.7: totality condition (TC).

$M$ satisfies $T C<=>\forall \varphi(\forall v \in W(R(w, v)=>M, v \mid=\varphi) \Rightarrow>\varphi E(w, 1))$. 
$T C$ states that a proposition that is true at all the worlds that are indistinguishable from $w$ is true in virtue of the essence of all the basic entities.

Theorem 2.3.3: consequences of $T C$.

(i) $M, w \mid=[X] \varphi=>[X] \varphi \in E(w, 1)$, by theorem 2.3.1 and $T C$.

(ii) $M, w \mid=\neg[X] \varphi \Rightarrow \neg[X] \varphi \in E(w, 1)$, by theorem 2.3.2 and $T C$.

(iii) $[X] \varphi \in E(w, V)$ or $\neg[X] \varphi \in E(w, 1)$, by (i) and (ii).

Theorem 2.3.4 (assuming TC): $M, w \mid=[X] \varphi=>[X] \varphi \in E(w,|\varphi| \cdot X)$.

$M, w \mid=[X] \varphi \Rightarrow[X] \varphi \in E(w, 1)$, by theorem 2.3.3. (i).

$M, w \mid=[X] \varphi \Rightarrow \neg[X] \varphi \notin E(w, 1)$, by $E 3$.

$M, w \mid=[X] \varphi \Rightarrow \neg[X] \varphi \notin E(w, \widetilde{s}(X \bullet|\varphi|)$, by $E 2$.

$M, w \mid=[X] \varphi \Rightarrow[X] \varphi \in E(w, \Im)(X \bullet|\varphi|)$, by $E 5$.

Theorem 2.3.5 (assuming $T C$ ): $M, w \mid=\neg[X] \varphi=>\neg[X] \varphi \in E(w,|\varphi| \cdot X)$.

$M, w \mid=\neg[X] \varphi \Rightarrow \neg[X] \varphi \in E(w, 1)$, by theorem 2.3.3. (ii).

$M, w \mid=\neg[X] \varphi=>[X] \varphi \notin E(w, 1)$, by $E 3$.

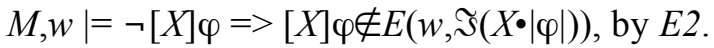

$M, w \mid=\neg[X] \varphi \Rightarrow \neg[X] \varphi \in E(w, \Im(X \bullet|\varphi|))$, by $E 5$.

\subsection{Soundness}

Soundness is proved by induction on the length of derivations.

\section{Theorem 2.4.1:}

E is sound with respect to the class of all models for $L(\mathbf{E})$ satisfying $T C$ and $U C$.

Axioms on delimiters.

Straightforward, by the following lemma and the definition of $\mathfrak{S}$.

Lemma 2.4.1: $\mathfrak{I}(X)=\Im(Y)=>M, w \mid=[X] \varphi \leftrightarrow[Y] \varphi$.

Indeed, $\mathfrak{\Im}(X)=\mathfrak{s}(Y)=>E(w, \mathfrak{s}(X))=E(w, \mathfrak{s}(Y))$, by the definition of $E$.

Hence, $\Im(X)=\Im(Y)=>(M, w|=[X] \varphi<=>M, w|=[Y] \varphi)$, i.e. $M, w \mid=[X] \varphi \leftrightarrow[Y] \varphi$.

Axioms on modalities:

M1: $\|-[X]\left(\varphi \rightarrow \varphi^{\prime}\right) \rightarrow\left([X] \varphi \rightarrow[X] \varphi^{\prime}\right)$.

Suppose $M, w \mid=[X]\left(\varphi \rightarrow \varphi^{\prime}\right)$ and $M, w \mid=[X] \varphi$.

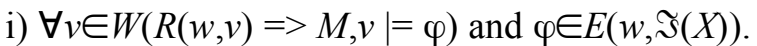

ii) $\forall v \in W\left(R(w, v)=>M, v \mid=\varphi \rightarrow \varphi^{\prime}\right)$ and $\left(\varphi \rightarrow \varphi^{\prime}\right) \in E(w, \Im(X))$.

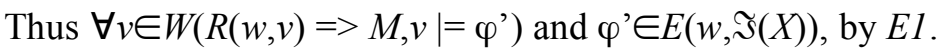


M2: $\|-[X] \varphi \rightarrow[X \cdot Y] \varphi$.

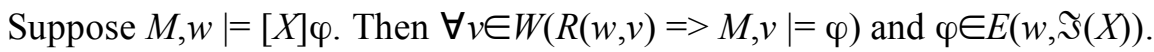

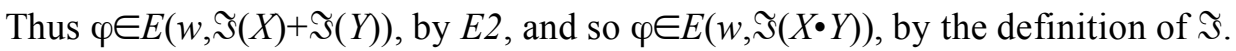

M3: $\|-[X] \varphi \rightarrow \varphi$.

Suppose $M, w \mid=[X] \varphi$. Then $\forall v \in W(R(w, v)=>M, v \mid=\varphi)$ and $\varphi \in E(w, \Im(X))$.

Thus $M, w \mid=\varphi$, since $R$ is reflexive by definition 2.3.1.

M4: $\|-[X] \varphi \rightarrow[X][X] \varphi$.

Suppose $M, w \mid=[X] \varphi$. Then $\forall v \in W(R(w, v)=>M, v \mid=\varphi)$ and $\varphi \in E(w, \Im(X))$.

Thus $\forall v \in W(R(w, v)=>M, v \mid=[X] \varphi)$, by theorem 2.3.1, and $[X] \varphi \in E(w, \Im(X))$, by $E 4$.

M5: $\|-[X \bullet|\varphi|][X] \varphi \vee[X \bullet|\varphi|] \neg[X] \varphi$.

$M, w \mid=[X] \varphi \Rightarrow \forall v \in W(R(w, v) \Rightarrow M, v \mid=[X] \varphi)$, by theorem 2.3.1.

$M, w \mid=[X] \varphi \Rightarrow[X] \varphi \in E(w, \Im(X \bullet|\varphi|))$, theorem 2.3.4.

Thus $M, w|=[X] \varphi=>M, w|=[X \bullet|\varphi|][X] \varphi$.

$M, w \mid=\neg[X] \varphi \Rightarrow \forall v \in W(R(w, v)=>M, v \mid=\neg[X] \varphi)$, by theorem 2.3.2.

$M, w \mid=\neg[X] \varphi \Rightarrow \neg[X] \varphi \in E(w, \Im(X \bullet|\varphi|))$, theorem 2.3.5.

Thus $M, w|=\neg[X] \varphi=>M, w|=\neg[X \bullet|\varphi|][X] \varphi$.

Therefore, $M, w \mid=[X \bullet|\varphi|][X] \varphi \vee[X \bullet|\varphi|] \neg[X] \varphi$.

M6: $[X] \varphi \wedge[Y \bullet|\varphi|] \varphi^{\prime} \rightarrow[Y \bullet X] \varphi^{\prime}$.

Suppose $M, w \mid=[X] \varphi$ and $M, w \mid=[Y \cdot|\varphi|] \varphi$ '.

i) $\forall v \in W(R(w, v)=>M, v \mid=\varphi)$ and $\varphi \in E(w, \Im(X))$.

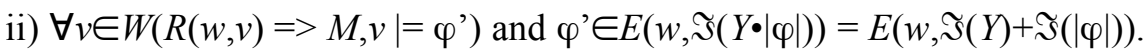

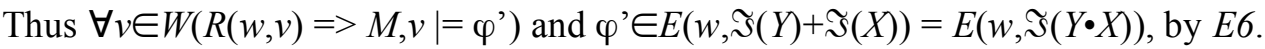

ME: $\|-\boldsymbol{E} \boldsymbol{x}(X)$.

Straightforward: $M, w \mid=\boldsymbol{E} \boldsymbol{x}(X)$ by the definition of truth.

RM: $\|-\varphi=>\|-[|\varphi|] \varphi$.

Suppose $\|-\varphi$. Then $\forall v \in W(R(w, v)=>M, w \mid=\varphi)$, and so $\varphi \in E(w, \Im(|\varphi|))$, by $U C$.

\subsection{Completeness}

Completeness follows from a canonical model construction. In this section, we will show how to construct a canonical model for $\mathbf{E}$ given a generic relation of ontological dependence. Then we will show how a certain relation of ontological dependence can be defined in $L(\mathbf{E})$.

\section{Theorem 2.5.1:}

$\mathbf{E}$ is strongly complete with respect to the class of all models for $L(\mathbf{E})$ satisfying $T C$ and $U C$. 
Definition 2.5.1: relation of ontological dependence.

Let $D$ be a relation between pluralities. Call $D$ a relation of ontological dependence if it satisfies the following set of conditions:

$\triangleright \mathbf{1}: \mid-X \triangleright X$

$\triangleright$ 2: $\mid-X \triangleright Y \wedge Y \triangleright Y^{\prime} \rightarrow X \triangleright Y^{\prime}$.

$\triangleright$ 3: $\mid-X \triangleright Y \rightarrow X \cdot Y^{\prime} \triangleright Y \cdot Y^{\prime}$.

$\triangleright \mathbf{4 :} \mid-X \triangleright Y \wedge[Y] \varphi \rightarrow[X] \varphi$.

$\triangleright \mathbf{R}:$ if for all $\varphi, \mid-[Y] \varphi \rightarrow[X] \varphi$, then $\mid-X \triangleright Y$.

Intuitively, $X \triangleright Y$ states that the $X$ depend on the $Y$, i.e., for every entity $y$ in $Y$ there is an entity $x$ in $X$ such that $x$ depends on $y$.

Proposition 2.5.1: let $\triangle$ be a relation of ontological dependence.

Then there is a canonical constituency model based on $D$.

The canonical constituency model based on $\triangleright$ is the tuple $B_{\mathbf{E}}=\left(B_{\mathbf{E}},{ }_{\mathbf{E}}, l_{\mathbf{E}}, \widetilde{\Im}_{\mathbf{E}}\right)$, where

$B_{\mathbf{E}}=\left\{[X]_{\mathbf{E}} \mid X\right.$ a delimiter $\}$, where $[X]_{\mathbf{E}}=\{Y||-X \triangleright Y\}$;

$+_{\mathbf{E}}$ is such that $[X]_{\mathbf{E}}+_{\mathbf{E}}[Y]_{\mathbf{E}}=[X \cdot Y]_{\mathbf{E}}$ and $l_{\mathbf{E}}=[V]_{\mathbf{E}}$;

$\mathfrak{I}_{\mathbf{E}}$ is such that $\mathfrak{I}_{\mathbf{E}}(X)=[X]_{\mathbf{E}}$.

Note that $\mathfrak{\Im} 1$ and $\mathfrak{s} 2$ are satisfied by definition, while $\mathfrak{\Im} 3$ follows from axioms D5-D8.

Fact 1: $Y \in[X]_{\mathbf{E}}<=>[Y]_{\mathbf{E}} \subseteq[X]_{\mathbf{E}}$.

Suppose $Y \in[X]_{\mathbf{E}}$ and $U \in[Y]_{\mathbf{E}}$.

Then $\mid-X \triangleright Y$ and $\mid-Y \triangleright U$.

Thus $\mid-X \triangleright U$, by $\triangleright \mathbf{2}$, and so $U \in[X]_{\mathbf{E}}$.

Suppose $[Y]_{\mathbf{E}} \subseteq[X]_{\mathbf{E}}$. Since $Y \in[Y]_{\mathbf{E}}$, by $\triangleright \mathbf{1}, Y \in[X]_{\mathbf{E}}$.

Fact 2: $[X]_{\mathbf{E}} \subseteq\left[X^{\prime}\right]_{\mathbf{E}}$ and $[Y]_{\mathbf{E}} \subseteq\left[Y^{\prime}\right]_{\mathbf{E}}=>[X \cdot Y]_{\mathbf{E}} \subseteq\left[X^{\prime} \cdot Y^{\prime}\right]_{\mathbf{E}}$.

Suppose $[X]_{\mathbf{E}} \subseteq\left[X^{\prime}\right]_{\mathbf{E}}$ and $[Y]_{\mathbf{E}} \subseteq\left[Y^{\prime}\right]_{\mathbf{E}}$.

Then $X \in\left[X^{\prime}\right]_{\mathbf{E}}$ and $Y \in\left[Y^{\prime}\right]_{\mathbf{E}}$, so that $\mid-X^{\prime} \triangleright X$ and $\mid-Y^{\prime} \triangleright Y$.

Thus $\mid-X^{\prime} \cdot Y \triangleright X \cdot Y$, by $\triangleright \mathbf{3}$, and $\mid-X^{\prime} \cdot Y^{\prime} \triangleright X \cdot Y$, by $\triangleright \mathbf{3}$ again.

Therefore $X \cdot Y \in\left[X^{\prime} \bullet Y^{\prime}\right]_{\mathbf{E}}$, and so $[X \bullet Y]_{\mathbf{E}} \subseteq\left[X^{\prime} \bullet Y^{\prime}\right]_{\mathbf{E}}$, by fact 1 .

Corollary: ${ }_{\mathbf{E}}$ is well defined, i.e. $[X]_{\mathbf{E}}=\left[X^{\prime}\right]_{\mathbf{E}}$ and $[Y]_{\mathbf{E}}=\left[Y^{\prime}\right]_{\mathbf{E}}=>\left[X^{\bullet} Y\right]_{\mathbf{E}}=\left[X^{\prime} \bullet Y^{\prime}\right]_{\mathbf{E}}$.

Fact 3: $+_{\mathbf{E}}$ satisfies conditions $+1,+2,+3,+4$.

This follows from the definition of $+_{\mathbf{E}}, \triangleright \mathbf{R}$ and axioms D1-D4.

Let us check $+1:[X]_{\mathbf{E}}=[X]_{\mathbf{E}}{ }_{\mathbf{E}}[Y]_{\mathbf{E}}$, i.e. $[X]_{\mathbf{E}}=[X \cdot X]_{\mathbf{E}}$.

$-[X] \varphi \leftrightarrow[X \cdot X] \varphi$, by D1. 
$-[X] \varphi \rightarrow[X \cdot X] \varphi$ and $\mid-[X \cdot X] \varphi \rightarrow[X] \varphi$.

Thus $\mid-X \triangleright X \cdot X$ and $\mid-X \cdot X \triangleright X$, by $\triangleright \mathbf{R}$.

$X \cdot X \in[X]_{\mathbf{E}}$ and $X \in[X \cdot X]_{\mathbf{E}}$, by the definition of $[X]_{\mathbf{E}}$, so that $[X]_{\mathbf{E}}=[X \cdot X]_{\mathbf{E}}$, by fact 1 .

Proposition 2.5.2: let $\triangle$ be a relation of ontological dependence.

Then there is a canonical model for $L(\mathbf{E})$ based on $\triangleright$.

The canonical model for $L(\mathbf{E})$ is the tuple $M_{\mathbf{E}}=\left(W_{\mathbf{E}}, R_{\mathbf{E}}, \mathrm{B}_{\mathbf{E}}, E_{\mathbf{E}}, V_{\mathbf{E}}\right)$, where

i) $W_{\mathbf{E}}$ is the set of maximal $\mathbf{E}$-consistent sets.

ii) $R_{\mathbf{E}}$ is such that $R_{\mathbf{E}}(w, v)<=>w^{*} \subseteq v$, where $w^{*}=\{\varphi \mid[V] \varphi \in w\}$.

iii) $\mathrm{B}_{\mathrm{E}}$ is the canonical constituency model induced by $D$.

iv) $E_{\mathbf{E}}$ is such that $E_{\mathbf{E}}\left(w,[X]_{\mathbf{E}}\right)=w / X$, where $w / X=\{\varphi \mid[X] \varphi \in w\}$.

v) $V_{\mathbf{E}}$ is such that $V_{\mathbf{E}}(p)=\{w \mid p \in w\}$.

Note that $E_{\mathbf{E}}$ is well defined, since $[X]_{\mathbf{E}}=[Y]_{\mathbf{E}}=>w / X=w / Y$, by $\triangleright \mathbf{4}$.

Note also that $w / X \subseteq w^{*}$ for every $X$, by $\mathbf{T} 1$.

Lemma 2.5.1: $w^{*} \mid-\varphi=>\varphi \in w^{*}$, i.e. $w^{*}$ is E-closed.

Suppose $w^{*} \mid-\varphi$. Then, there are $\varphi_{1}, \ldots, \varphi_{\mathrm{n}}$ such that $\varphi_{1}, \ldots, \varphi_{\mathrm{n}} \mid-\varphi$ and $[V] \varphi, \ldots,[V] \varphi \in w$.

Thus, $[V] \varphi_{1}, \ldots,[V] \varphi_{\mathrm{n}} \mid-[V] \varphi$, by $\mathbf{T} 7$, and so $[V] \varphi \in w$ and $\varphi \in w^{*}$.

Lemma 2.5.2: $\forall v \in W_{1}\left(w^{*} \subseteq v=>\varphi \in v\right)=>\varphi \in w^{*}$.

Suppose $\forall v \in W_{1}\left(w^{*} \subseteq v=>\varphi \in v\right)$. Then, there is no set $v$ such that $w^{*} \subseteq v$ and $\neg \varphi \in v$.

Thus, $w^{*} \cup\{\neg \varphi\}$ is not consistent; $w^{*} \mid-\varphi$, and so $\varphi \in w^{*}$, by lemma 2.5.1.

Lemma 2.5.3: $w^{*} \subseteq v=>w / X=v / X$.

Suppose $w^{*} \subseteq v$ and $\varphi \in w / X ;[X] \varphi \in w / X \bullet|\varphi|$, by T2, $[X] \varphi \in w^{*}$, by T1; $\varphi \in v / X$.

Suppose $w^{*} \subseteq v$ and $\varphi \notin w / X ; \neg[X] \varphi \in w / X \bullet|\varphi|$, by T3; $\neg[X] \varphi \in w^{*}$, by T1; $\varphi \notin v / X$.

Lemma 2.5.4: $w^{*} \subseteq v=>w^{*}=v^{*}$.

Suppose $w^{*} \subseteq v$ and $\varphi \in w^{*} ; \varphi \in w / V ; \varphi \in v / V$, by lemma 2.5.3, $\varphi \in v^{*}$.

Suppose $w^{*} \subseteq v$ and $\varphi \notin w^{*} ; \varphi \notin w / V ; \varphi \notin v / V$, by lemma 2.5.3, $\varphi \notin v^{*}$.

Lemma 2.5.5: $\left(W_{\mathbf{E}}, R_{\mathbf{E}}, \mathbf{B}_{\mathbf{E}}, E_{\mathbf{E}}\right)$ is a frame for $L(\mathbf{E})$.

$R_{\mathbf{E}}$ is an equivalence relation.

It suffices to show that $w^{*} \subseteq w$ and $w^{*} \subseteq v=>w^{*}=v^{*}$ :

$w^{*} \subseteq w$ follows from $\mathbf{M 3}$ and $w^{*} \subseteq v \Rightarrow w^{*}=v^{*}$ holds by lemma 2.5.4.

$E_{\mathbf{E}}$ satisfies conditions $E 1-E S$. 
E1-E6 follow from M1-M6.

$E R:[X] \varphi \in E_{\mathbf{E}}(w, Y)=>\varphi \in E_{\mathbf{E}}(w, Y)$ holds by $\mathbf{T 6}$.

$E S: R_{\mathbf{E}}(w, v)=>E_{\mathbf{E}}(w, X)=E_{\mathbf{E}}(v, X)$ holds by lemma 2.5.3.

Truth Lemma: $M_{\mathbf{1}}, w \mid=\varphi<=>\in w$.

The proof is by induction. The only non-standard cases are the following ones.

Case 1: $M_{\mathbf{E}}, w \mid=\boldsymbol{E} \boldsymbol{x}(X)<=>\boldsymbol{E} \boldsymbol{x}(X) \in w$.

Straightforward, by ME.

Case 2: $M_{\mathbf{E}}, w \mid=[X] \varphi<=>[X] \varphi \in w$.

$M_{\mathbf{E}}, w \mid=[X] \varphi \Leftrightarrow \forall v \in W_{\mathbf{E}}\left(R_{\mathbf{E}}(w, v) \Rightarrow M_{\mathbf{E}}, v \mid=\varphi\right)$ and $\varphi \in E_{\mathbf{E}}(w, \Im(X))$.

$M_{\mathbf{E}}, w \mid=[X] \varphi<=>\forall \in W_{\mathbf{E}}\left(w^{*} \subseteq v=>\varphi \in v\right)$ and $\varphi \in w / X$, by I.H.

$M_{\mathbf{E}}, w \mid=[X] \varphi<=\varphi \in w / X$, since $w / X \subseteq w^{*}$.

$M_{\mathbf{E}}, w \mid=[X] \varphi \Leftrightarrow[X] \varphi \in w$.

Lemma 2.5.6: $M_{\mathrm{E}}$ satisfies the totality condition (TC).

Suppose $\forall v \in W_{\mathbf{E}}\left(R_{\mathbf{E}}(w, v)=>M_{\mathbf{E}}, v \mid=\varphi\right)$, i.e. $\forall v \in W_{\mathbf{E}}\left(w^{*} \subseteq v=>\varphi \in v\right)$.

Then $\varphi \in w^{*}$, by 2.5.1, and so $\varphi \in w / V$, by the definitions of $w^{*}$ and $w / V$.

Lemma 2.5.7: $M_{\mathbf{E}}$ satisfies the universality condition (UC).

Suppose $\forall w \in W_{\mathbf{E}}\left(M_{\mathbf{E}}, w \mid=\varphi\right)$. Then $\forall w \in W_{\mathbf{E}}(\varphi \in w)$, by the truth lemma. Thus $\mid-\varphi$, by the definition of $W_{\mathbf{E}}$. Therefore $\mid-[|\varphi|] \varphi$, by $\mathbf{R M}$, and so $\varphi \in w /|\varphi|=E(w, \Im(|\varphi|))$, for every $w \in W$.

In conclusion, $M_{1}$ is a model for $L(\mathbf{E})$ satisfying $T C$ and $U C$.

All we have to show now is that an ontological dependence relation is definable in $L(\mathbf{E})$.

Definition 2.5.2: relation of dependence induced by $\boldsymbol{E x}$.

Let $\triangleright$ be the relation defined by $X \triangleright Y:=[X] \boldsymbol{E} \boldsymbol{x}(Y)$. Then $\triangleright$ is a relation of dependence.

$\triangleright \mathbf{1}: \mid-X \triangleright X$

$\mid-[|\boldsymbol{E x}(X)|] \boldsymbol{E x}(X)$, by ME and RM.

$-[X] \boldsymbol{E} \boldsymbol{x}(X)$, by $\mathbf{D} 7$.

$\triangleright$ 2: $\mid-X \triangleright Y \wedge Y \triangleright Z \rightarrow X \triangleright Z$.

$-[X] \boldsymbol{E} \boldsymbol{x}(Y) \wedge[|\boldsymbol{E} \boldsymbol{x}(Y)|] \boldsymbol{E} \boldsymbol{x}\left(Y^{\prime}\right) \rightarrow[X] \boldsymbol{E} \boldsymbol{x}\left(Y^{\prime}\right)$, by M6.

$-[X] \boldsymbol{E} \boldsymbol{x}(Y) \wedge[Y] \boldsymbol{E} \boldsymbol{x}\left(Y^{\prime}\right) \rightarrow[X] \boldsymbol{E x}\left(Y^{\prime}\right)$, by $\mathbf{D} 7$.

$\triangleright$ 3: $\mid-X \triangleright Y \rightarrow X \cdot Y^{\prime} \triangleright Y \cdot Y^{\prime}$.

$-[X] \boldsymbol{E x}(Y) \wedge\left[Y \bullet Y^{\prime}\right] \boldsymbol{E x}\left(Y \bullet Y^{\prime}\right) \rightarrow\left[X \cdot Y^{\prime}\right] \boldsymbol{E x}\left(Y \bullet Y^{\prime}\right)$, by $\mathbf{T 5}$.

$-[X] \boldsymbol{E x}(Y) \rightarrow\left[X \cdot Y^{\prime}\right] \boldsymbol{E} \boldsymbol{x}\left(Y \bullet Y^{\prime}\right)$, by $\triangleright \mathbf{1}$. 
$\triangleright \mathbf{4 :} \mid-X \triangleright Y \wedge[Y] \varphi \rightarrow[X] \varphi$.

$\mid-[X] \boldsymbol{E} \boldsymbol{x}(Y) \wedge[|\boldsymbol{E} \boldsymbol{x}(Y)|] \varphi \rightarrow[X] \varphi$, by M6.

$-[X] \boldsymbol{E} \boldsymbol{x}(Y) \wedge[Y] \varphi \rightarrow[X] \varphi$, by $\mathbf{D} 7$.

$\triangleright \mathbf{R}:|-[Y] \varphi \rightarrow[X] \varphi=>|-X \triangleright Y$.

Suppose $\mid-[Y] \varphi \rightarrow[X] \varphi$, for all $\varphi$.

Then $\mid-[Y] \boldsymbol{E} \boldsymbol{x}(Y) \rightarrow[X] \boldsymbol{E} \boldsymbol{x}(Y)$, and so $\mid-[X] \boldsymbol{E} \boldsymbol{x}(Y)$, by $\triangleright \mathbf{1}$.

This concludes the proof of theorem 2.5.1.

\subsection{Essence and dependence}

A version $\mathbf{E D}$ of system $\mathbf{E}$, based on the notion of dependence, can be defined as follows ${ }^{7}$. The alphabet of the language, $L(\mathbf{E D})$, is like $L(\mathbf{E})$, with the exception that, instead of the existence predicate, it contains the ontological dependence relation $\triangle$ between terms. The definition of formula is changed accordingly: if $X, Y \in \operatorname{Tm}(\mathbf{E D})$, then $(X \triangleright Y) \in F m(\mathbf{E D})$. ED is characterized by the following groups of axioms and rules.

Group I: classical axioms and modus ponens.

Group II: modal axioms and rules.

\begin{tabular}{|l|l|}
\hline Axioms on dependence & Definition: co-dependence. \\
\hline$\triangleright \mathbf{1 :} X \triangleright X$. & $X \approx Y:=X \triangleright Y \wedge Y \triangleright X$. \\
$\triangleright \mathbf{2 :} X \triangleright Y \wedge Y \triangleright Y^{\prime} \rightarrow X \triangleright Y^{\prime}$. & \\
$\triangleright \mathbf{3}: X \triangleright Y \rightarrow X^{\prime} Y^{\prime} \triangleright Y \bullet Y^{\prime}$. & \\
$\triangleright \mathbf{4 :} X \triangleright Y \wedge[Y] \varphi \rightarrow[X] \varphi$. & Axioms on modalities \\
\hline Axioms on delimiters & M1: $[X]\left(\varphi \rightarrow \varphi^{\prime}\right) \wedge[X] \varphi \rightarrow[X] \varphi^{\prime}$. \\
\hline DD1: $X \approx X \bullet X$. & M2: $[X] \varphi \rightarrow[X \bullet Y] \varphi$. \\
DD2: $X \bullet Y \approx Y \bullet X$. & M3: $[X] \varphi \rightarrow \varphi$. \\
DD3: $X \bullet\left(Y \bullet Y^{\prime}\right) \approx\left[(X \bullet Y) \bullet Y^{\prime}\right.$. & M4: $[X] \varphi \rightarrow[X][X] \varphi$. \\
DD4: $X \bullet V \approx V$. & M5: $[X \bullet|\varphi|][X] \varphi \vee[X \bullet|\varphi|] \neg[X] \varphi$. \\
DD5: $|\neg \varphi| \approx|\varphi|$. & M6: $[X] \varphi \wedge[Y \bullet|\varphi|] \varphi \rightarrow[Y \bullet X] \varphi^{\prime}$. \\
DD6: $\left|\varphi \wedge \varphi^{\prime}\right| \approx|\varphi| \bullet\left|\varphi^{\prime}\right|$. & MD: $[X] \varphi \rightarrow X \triangleright|\varphi|$. \\
DD7: $|X \triangleright Y| \approx X$. & RM: $|-\varphi=>|-[|\varphi|] \varphi$. \\
DD8: $|[X] \varphi| \approx X \bullet|\varphi|$. &
\end{tabular}

\footnotetext{
${ }^{7}$ The following system can be seen as the propositional version of the quantified logic of essence proposed in [5].
} 
ED can be interpreted in $\mathbf{E}$ by defining $X \triangleright Y:=[X] \boldsymbol{E} \boldsymbol{x}(Y)$ as in section 2.5. DD1-DD8 are straightforward consequences of D1-D8 and ME, while MD is derivable as follows:

$\mid-[X] \varphi \wedge[|\varphi|] \boldsymbol{E x}(|\varphi|) \rightarrow[X] \boldsymbol{E x}(|\varphi|)$, by M6.

$-[X] \varphi \rightarrow[X] \boldsymbol{E x}(|\varphi|)$, by ME and $\mathbf{R M}$.

Conversely, it is not difficult to see that $\mathbf{E}$ can be interpreted in ED by putting $\boldsymbol{E} \boldsymbol{x}(X):=X \triangleright X$. Indeed, D1-D8 follow from DD1-DD8 and $\triangleright 4$, and ME is a consequence of $\triangleright \mathbf{1}$.

\subsection{Essence and necessity}

In [3] we find the following statement:

Indeed, it seems to me that far from viewing essence as a special case of metaphysical necessity, we should view metaphysical necessity as a special case of essence. For each class of objects, be they concepts or individuals or entities of some other kind, will give rise to its own domain of necessary truths, the truths which flow from the nature of the objects in question. The metaphysically necessary truths can then be identified with the propositions which are true in virtue of the nature of all objects whatever. ( $p .9)$

Let us consider the following condition on models of $\mathbf{E}$.

Definition 2.7.1: necessity condition (NC).

$M$ satisfies $N C<=\forall \varphi \forall w \in W(\varphi \in E(w, X) \Rightarrow \forall \forall \in W(R(w, v)=>M, v \mid=\varphi))$.

Since $\mathbf{E}$ is sound with respect to the class of models satisfying $T C$ and $U C$, it is also sound with respect to the class of models satisfying $T C, U C$, and $N C$. We now prove the following:

Theorem 2.7.1: $\mathbf{E}$ is strongly complete with respect to the class of all models satisfying $T C, U C$ and $N C$.

It suffices to show that the canonical model for $\mathbf{E}$ satisfies $N C$.

Suppose $\varphi \in w / X$. Then $\varphi \in w^{*}$, and so $\forall v \in W_{\mathbf{1}}\left(w^{*} \subseteq v=>\varphi \in v\right)$.

If we say that a proposition is necessarily true at $w$ if and only if it is true at every world that is accessible from $w$, then the set of all propositions that are necessarily true at $w$ coincides with the set of all propositions that are true at $w$ in virtue of the essence of all basic entities. As a consequence, we can introduce the following definition.

Definition 2.7.2: necessary truth.

Let $\square \varphi$ state that $\varphi$ is a necessary truth. $\square \varphi:=[V] \varphi$. 
Since $[V] \varphi$ is true at $w$ if and only if there exists an $X$ such that $[X] \varphi$ is true at $w$, one can conclude that essential truths are the source of necessary truths.

\section{System $\mathbf{E}^{=}$}

In this section it will be shown that the system proposed in [2] can be interpreted along the lines presented in the previous section.

The alphabet of the language $L\left(\mathbf{E}^{=}\right)$of $\mathbf{E}^{=}$contains the following signs ${ }^{8}$ :

- a countable set $P V$ of variables for propositions $\left(p, p^{\prime}, p^{\prime \prime}, \ldots\right)$.

- a countable set $D V$ of variables for delimiters $\left(d, d^{\prime}, d^{\prime \prime}, \ldots\right)$.

- a constant for the universal delimiter: $V$.

- the composition operator: $\bullet$.

- the constitution operator: ||.

- the closure operator: $\mathbf{c}$.

- the predicate of identity: $=$.

- the modality constructor: [ ].

- brackets: ( and ).

The sets $\operatorname{Tm}\left(\mathbf{E}^{\mathbf{}}\right)$ and $F m\left(\mathbf{E}^{\mathbf{}}\right)$ of terms and formulas of $L\left(\mathbf{E}^{-}\right)$are defined by simultaneous induction according to the following formation rules.

Rules for terms:

$X:=d|V| X \cdot Y|\mathbf{c} X||\varphi| \quad$ where $d \in D V$ and $\varphi \in F m\left(\mathbf{E}^{=}\right)$

Rules for formulas:

$\varphi:=p|\neg \varphi|(\varphi \wedge \varphi)|X=Y|[X] \varphi \quad$ where $p \in P V$ and $X, Y \in \operatorname{Tm}\left(\mathbf{E}^{=}\right)$

A formula like $X=Y$ states that the pluralities $X$ and $Y$ are identical. The closure operator is interpreted as the operation that takes a plurality $X$ as input and returns the plurality of all the entities on which $X$ depends as output. As a consequence, a formula like $Y \cdot \mathbf{c} X=\mathbf{c} X$ states that $Y$ is included in the closure of $X$, i.e. that $X$ depends on $Y .^{9}$

\subsection{Axioms}

System $\mathbf{E}^{=}$is determined by the following groups of axioms and rules.

Group I: classical axioms and modus ponens.

\footnotetext{
${ }^{8}$ This is the system of propositional logic of essence proposed in [2].

${ }^{9}$ See [2], section I, for a more detailed description of the basic concepts.
} 
Group II: modal axioms and rules.

\begin{tabular}{|c|c|}
\hline Axioms on delimiters & Axioms on modalities \\
\hline $\begin{array}{l}\mathbf{I}^{=} \mathbf{1}: X=X \\
\mathbf{I}^{=} \mathbf{2}: X=Y \wedge X=Y^{\prime} \rightarrow Y=Y^{\prime} \\
\mathbf{I}^{=} \mathbf{3}: X=Y \rightarrow X \cdot Y^{\prime}=Y \cdot Y^{\prime} \\
\mathbf{I}^{=} \mathbf{4}: X=Y \wedge[X] \varphi \rightarrow[Y] \varphi\end{array}$ & \multirow{3}{*}{$\begin{array}{l}\text { M1: }[X]\left(\varphi \rightarrow \varphi^{\prime}\right) \wedge[X] \varphi \rightarrow[X] \varphi^{\prime} . \\
\text { M2: }[X] \varphi \rightarrow[X \bullet Y] \varphi . \\
\text { M3: }[X] \varphi \rightarrow \varphi . \\
\text { M5: }[X \bullet|\varphi|][X] \varphi \vee[X \bullet|\varphi|] \neg[X] \varphi . \\
\text { MI: }[X](X=Y) \vee[X] \neg(X=Y) \\
\text { RM: }|-\varphi=>|-[|\varphi|] \varphi .\end{array}$} \\
\hline $\begin{array}{l}\mathbf{D}^{\mathbf{}} \mathbf{1}: X \cdot X=X \\
\mathbf{D}^{=} \mathbf{2}: X \cdot Y=Y \cdot X \\
\mathbf{D}^{=} \mathbf{3}: X \cdot\left(Y \cdot Y^{\prime}\right)=(X \cdot Y) \cdot Y^{\prime} \\
\mathbf{D}^{=} \mathbf{4}: X \cdot V=V\end{array}$ & \\
\hline $\begin{array}{l}\mathbf{D}^{\mathbf{}} \mathbf{5}:|\neg \varphi|=|\varphi| \\
\mathbf{D}^{\mathbf{}} \mathbf{6}:\left|\varphi \wedge \varphi^{\prime}\right|=|\varphi| \cdot\left|\varphi^{\prime}\right| \\
\mathbf{D}^{=} \mathbf{7}:|X=Y|=X \cdot Y \\
\mathbf{D}^{=} \mathbf{8}:|[X] \varphi|=X \cdot|\varphi|\end{array}$ & \\
\hline $\begin{array}{l}D^{\mathbf{c}} \mathbf{1}: X \cdot \mathbf{c} X=\mathbf{c} X \\
\mathbf{D}^{\mathbf{c}} \mathbf{2}: X=Y \rightarrow \mathbf{c} X=\mathbf{c} Y \\
\mathbf{D}^{\mathbf{c}} \mathbf{3}: \mathbf{c} X=\mathbf{c c} X \\
\mathbf{D}^{\mathbf{c}} \mathbf{4}: \mathbf{c}(X \cdot Y)=\mathbf{c} X \cdot \mathbf{c} Y\end{array}$ & $\begin{array}{l}\mathbf{M}^{\mathbf{c}} \mathbf{1}:[\mathbf{c} X] \varphi \rightarrow[X] \varphi \\
\mathbf{M}^{\mathbf{c} 2:}[X] \varphi \rightarrow|\varphi| \cdot \mathbf{c} X=\mathbf{c} X\end{array}$ \\
\hline
\end{tabular}

\subsection{Theorems}

M4 and M6 are derivable in $\mathbf{E}^{=}$.

$\mathbf{T}^{\mathbf{}} \mathbf{1}: \mid-[X] \varphi \rightarrow[X][X] \varphi$.

$|-| \varphi \mid \cdot \mathbf{c} X=\mathbf{c} X \wedge[|\varphi| \cdot \mathbf{c} X][X] \varphi \rightarrow[\mathbf{c} X][X] \varphi$, by $\mathbf{I}^{\mathbf{*}} \mathbf{4}$.

$|-| \varphi \mid \cdot \mathbf{c} X=\mathbf{c} X \wedge[|\varphi| \cdot X][X] \varphi \rightarrow[\mathbf{c} X][X] \varphi$, by M2.

$\mid-[X] \varphi \wedge[|\varphi| \cdot X][X] \varphi \rightarrow[\mathbf{c} X][X] \varphi$, by $\mathbf{M}^{\mathbf{c}} \mathbf{2}$.

$-[X] \varphi \wedge[|\varphi| \cdot X][X] \varphi \rightarrow[X][X] \varphi$, by $\mathbf{M}^{\mathbf{c}} \mathbf{1}$.

$-[X] \varphi \rightarrow[|\varphi| \cdot X][X] \varphi$, by M3 and M5.

$-[X] \varphi \rightarrow[X][X] \varphi$, by pr. logic.

$\mathbf{T}^{=} \mathbf{2}: \mid-[X] \varphi \wedge[|\varphi|] \varphi^{\prime} \rightarrow[X] \varphi^{\prime}$.

$|-| \varphi \mid \cdot \mathbf{c} X=\mathbf{c} X \wedge[|\varphi| \cdot \mathbf{c} X] \varphi^{\prime} \rightarrow[\mathbf{c} X] \varphi^{\prime}$, by $\mathbf{I}^{\mathbf{}} \mathbf{4}$.

$|-| \varphi \mid \cdot \mathbf{c} X=\mathbf{c} X \wedge[|\varphi|] \varphi^{\prime} \rightarrow[\mathbf{c} X] \varphi^{\prime}$, by $\mathbf{M 2}$.

$\mid-[X] \varphi \wedge[|\varphi|] \varphi^{\prime} \rightarrow[\mathbf{c} X] \varphi^{\prime}$, by $\mathbf{M}^{\mathbf{c}} \mathbf{2}$.

$\mid-[X] \varphi \wedge[|\varphi|] \varphi^{\prime} \rightarrow[X] \varphi^{\prime}$, by $\mathbf{M}^{\mathbf{c}} \mathbf{1}$.

Furthermore, 
$\mathbf{T}^{\mathbf{}} \mathbf{3}: \mid-X \cdot Y=Y \wedge[X] \varphi \rightarrow[Y] \varphi$.

$\mid-X \cdot Y=Y \wedge[X \cdot Y] \varphi \rightarrow[Y] \varphi$, by $\mathbf{I}^{\mathbf{4}} \mathbf{4}$.

$-X \cdot Y=Y \wedge[X] \varphi \rightarrow[Y] \varphi$, by $\mathbf{M 2}$.

$\mathbf{T}^{=} \mathbf{4}: \mid-[X] \varphi \leftrightarrow[\mathbf{c} X] \varphi$.

$-[X] \varphi \rightarrow[\mathbf{c} X] \varphi$, by $\mathbf{D}^{\mathbf{c}} \mathbf{1}$ and $\mathbf{T} \mathbf{9}$.

$-[\mathbf{c} X] \varphi \rightarrow[X] \varphi$, by $\mathbf{M}^{\mathbf{c}} \mathbf{1}$.

Finally, $\mathbf{E}$ is interpretable in $\mathbf{E}^{=}$.

Let us define (EX): $\boldsymbol{E x}(X) \leftrightarrow X=X$. Then $\left.\right|_{-\mathbf{E}} \varphi=>\left.\right|_{\mathbf{E X}} \varphi\left(||_{\mathbf{E X}}=\right.$ derivability in $\left.\mathbf{E}^{=}+\mathbf{E X}\right)$.

Indeed, it is not difficult to see that axioms D1-D8 are derivable from $\mathbf{D}^{\mathbf{}} \mathbf{1}-\mathbf{D}^{=} \mathbf{8}$ and $\mathbf{I}^{=} \mathbf{4}$.

\subsection{Semantics}

Definition 3.3.1: constituency $\gamma$-structure.

A constituency $\gamma$-structure is a joint semilattice with one and the closure operator, $\boldsymbol{B}=(B,+, 1, \gamma)$. Hence, $\gamma$ is a monadic operation on $B$ satisfying:
i) $b \leq \gamma(b)$.
ii) $b \leq b^{\prime}=\gamma(b) \leq \gamma\left(b^{\prime}\right)$.
iii) $\gamma(\gamma(b))=\gamma(b)$.
iv) $\gamma\left(b+b^{\prime}\right)=\gamma(b)+\gamma\left(b^{\prime}\right)$.

Note that ii) actually follows from iv):

$b \leq b^{\prime}<=>b+b^{\prime}=b^{\prime}=>\gamma\left(b+b^{\prime}\right)=\gamma\left(b^{\prime}\right)<=>\gamma(b)+\gamma\left(b^{\prime}\right)=\gamma\left(b^{\prime}\right)<=>\gamma(b) \leq \gamma\left(b^{\prime}\right)$.

Definition 3.3.2: constituency $\gamma$-model.

A constituency $\gamma$-model is a pair $(\boldsymbol{B}, \mathfrak{\Im})$ defined as in section 2.3 such that

I3: $\Im(\mathbf{c} X)=\gamma(\mathfrak{I}(X))$

Definition 3.3.3: frame for $L\left(\mathbf{E}^{\mathbf{E}}\right)$.

A frame for $L\left(\mathbf{E}^{-}\right)$is a tuple $(W, R, \mathrm{~B}, E)$, where

i) $W$ is the set of possible worlds.

ii) $R \subseteq W \times W$ is an equivalence relation on $W$.

iii) $\mathrm{B}$ is a function assigning a $\gamma$-model $\mathrm{B}(w)=\left(\boldsymbol{B}_{w}, \mathfrak{\Im}_{w}\right)$ to each possible world.

iv) $E: W \times B \rightarrow \wp(F m(\mathbf{E}))$ is a selection function respecting the following conditions:

E1-ES.

$E=: \mathfrak{I}(X)=\mathfrak{\Im}(Y) \Rightarrow(X=Y) \in E(w, \mathfrak{I}(X))$. 
$E C 1: E(w, b)=E(w, \gamma(b))$.

$E C 2: \varphi \in E(w, b)=>\Im(|\varphi|)+\gamma(b)=\gamma(b)$.

In addition, $(W, R, \mathrm{~B}, E)$ has to satisfy the condition $R B: R(w, v)=>\mathrm{B}(w)=\mathrm{B}(v)$.

$R B$ states that the same constituency models are assigned to worlds that are $R$-indistinguishable.

Definition 3.3.4: model for $L\left(\mathbf{E}^{\mathbf{E}}\right)$.

A model for $L\left(\mathbf{E}^{=}\right)$is a tuple $M=(W, R, \mathrm{~B}, E, V)$, where $V: P V \rightarrow \wp(W)$ is a modal valuation.

Definition 3.3.5: truth (in a model at a world).

$M, w \mid=p<=>w \in V(p)$.

$M, w|=\neg \varphi<=>\operatorname{not} M, w|=\varphi$.

$M, w\left|=\varphi \wedge \varphi^{\prime}<=>M, w\right|=\varphi$ and $M, w \mid=\varphi^{\prime}$.

$M, w \mid=X=Y<=>\mathfrak{I}_{w}(X)=\mathfrak{I}_{w}(Y)$.

$M, w \mid=[X] \varphi<=\forall v \in W(R(w, v)=>M, v \mid=\varphi)$ and $\varphi \in E\left(w, \widetilde{I}_{w}(X)\right)$.

\subsection{Soundness}

Soundness is proved by induction on the length of derivations.

\section{Theorem 3.4.1:}

$\mathbf{E}^{-}$is sound with respect to the class of all models for $L\left(\mathbf{E}^{-}\right)$satisfying $T C$ and $U C$.

The validity of $\mathbf{I}^{=} \mathbf{1}-\mathbf{I}^{\mathbf{E}} \mathbf{4}$ and $\mathbf{D}^{\mathbf{E}} \mathbf{1}-\mathbf{D}^{=} \mathbf{8}$ is a straightforward consequence of the definition of $\mathfrak{I}$ and the definition of the truth of $X=Y$. The proof of the validity of the modal axioms is similar to the one presented in section 2. In particular, the validity of $\mathbf{M I}, \mathbf{M}^{\mathbf{c}} \mathbf{1}$ and $\mathbf{M}^{\mathbf{c}} \mathbf{2}$ follows without difficulty from conditions $E=, E C 1$ and $E C 2$.

\subsection{Completeness}

Completeness is proved by a canonical model construction.

\section{Theorem 3.5.1:}

$\mathbf{E}^{=}$is strongly complete with respect to the class of all models for $L\left(\mathbf{E}^{\mathbf{}}\right)$ satisfying $T C$ and $U C$.

Definition 3.5.1: canonical constituency $\gamma$-model.

Let $w$ be a maximal $\mathbf{E}^{=}$-consistent set of formulas.

The canonical constituency $\gamma$-model induced by $w$ is the tuple $\mathrm{B}_{\mathbf{w}}=\left(B_{\mathbf{w}},{ }_{\mathrm{w}}, 1_{\mathrm{w}}, \gamma_{\mathrm{w}}, \widetilde{I}_{\mathbf{w}}\right)$, where

$B_{\mathrm{w}}=\left\{[X]_{\mathrm{w}} \mid X\right.$ a delimiter $\}$, where $\left.[X]_{\mathrm{w}}=\{Y \mid(X=Y) \in w)\right\}$;

$+_{\mathrm{w}}$ is such that $[X]_{\mathrm{w}}+_{\mathrm{w}}[Y]_{\mathrm{w}}=[X \cdot Y]_{\mathrm{w}} ; 1_{\mathrm{w}}=[V]_{\mathrm{w}}$;

$\gamma_{\mathrm{w}}$ is such that $\gamma_{\mathrm{w}}\left([X]_{\mathrm{w}}\right)=[\mathbf{c} X]_{\mathrm{w}}$;

$\mathfrak{I}_{\mathbf{w}}$ is such that $\mathfrak{I}_{\mathrm{w}}(X)=[X]_{\mathrm{w}}$. 
Note that both $+_{w}$ and $\gamma_{w}$ are well defined by $\mathbf{I}^{=} \mathbf{1}-\mathbf{I}^{=} \mathbf{3}$ and $\mathbf{D}^{\mathbf{c}} \mathbf{2}$.

Fact 1: $(X=Y) \in w<=>Y \in[X]_{\mathrm{w}}<=>[X]_{\mathrm{w}}=[Y]_{\mathrm{w}}$.

Straightforward, by the definition of $[X]_{\mathrm{w}}$ and $\mathbf{I}^{\mathbf{}} \mathbf{1 - \mathbf { I }} \mathbf{2}$.

Fact 2: $+_{\mathrm{w}}$ satisfies conditions $+1,+2,+3,+4$.

Straightforward, by the definition of $+_{w}$ and $\mathbf{D}^{=} \mathbf{1 - D} \mathbf{4}$.

Fact 3: $\gamma_{w}$ satisfies conditions $\gamma 1, \gamma 2, \gamma 3, \gamma 4$.

Straightforward, by the definition of $\gamma_{w}$ and $\mathbf{D}^{\mathbf{c}} \mathbf{1}-\mathbf{D}^{\mathbf{c}} \mathbf{4}$.

Definition 3.5.2: canonical model for $L\left(\mathbf{E}^{\overline{ }}\right)$.

A canonical model for $L\left(\mathbf{E}^{-}\right)$is a tuple $M=(W, R, \mathrm{~B}, E, V)$, where

i) $W$ is the set of maximal $\mathbf{E}^{=}$-consistent sets.

ii) $R$ is such that $R(w, v)<=>w^{*} \subseteq v$, where $w^{*}=\{\varphi \mid[V] \varphi \in w\}$.

iii) $\mathrm{B}$ is such that $\mathrm{B}(w)=\mathrm{B}_{\mathbf{w}}$, for every $w \in W$.

iv) $E$ is such that $E\left(w,[X]_{\mathrm{w}}\right)=w / X$, where $w / X=\{\varphi \mid[X] \varphi \in w\}$.

v) $V$ is such that $V(p)=\{w \mid p \in w\}$.

Note that $E$ is well defined, by fact 1 and $\mathbf{I}^{\mathbf{4}} \mathbf{4}$ :

$[X]_{\mathbf{w}}=[Y]_{\mathbf{w}}<=>(X=Y) \in w<=>([X] \varphi \leftrightarrow[Y] \varphi) \in w<=>w / X=w / Y$.

Lemma 3.5.1: $(W, R, \mathrm{~B}, E)$ is a frame for $L\left(\mathbf{E}^{\mathbf{E}}\right)$.

1) $R$ is an equivalence relation: analogous to lemma 2.5.5.

2) $E$ satisfies conditions $E 1-E S$ : analogous to lemma 2.5.5.

3) $E$ satisfies conditions $E=, E C 1$ and $E C 2$ : by $\mathbf{M I}, \mathbf{T}^{\mathbf{}} \mathbf{4}$ and $\mathbf{M}^{\mathbf{c}} \mathbf{2}$.

4) $(W, R, \mathrm{~B}, E)$ satisfies conditions $R B: R(w, v)=\mathrm{B}(w)=\mathrm{B}(v)$.

Suppose $R(w, v)$, i.e. $w^{*} \subseteq v$. We have to show that $\left(B_{\mathbf{w}},+_{\mathbf{w}}, l_{\mathrm{w}}, \gamma_{\mathrm{w}}, \mathfrak{I}_{\mathbf{w}}\right)=\left(B_{\mathbf{v}},+_{\mathbf{v}}, l_{\mathrm{v}}, \gamma_{\mathbf{v}}, \mathfrak{I}_{\mathbf{v}}\right)$.

i) $(X=Y) \in w<=>(X=Y) \in w^{*}$.

$(X=Y) \in w<=>[X](X=Y) \in w$, by MI and T1.

$(X=Y) \in w<=>[V](X=Y) \in w$, by $\mathbf{T} 2$ and $\mathbf{T 1}$, and so $(X=Y) \in w<=>(X=Y) \in w^{*}$.

ii) $w^{*} \subseteq v \Rightarrow(X=Y) \in w<=>(X=Y) \in v$.

Suppose $w^{*} \subseteq v$. Then $w^{*}=v^{*}$, by lemma 2.5.4.

Thus $(X=Y) \in w^{*}<=>(X=Y) \in v^{*}$, and so $(X=Y) \in w<=>(X=Y) \in v$, by i)

iii) $w^{*} \subseteq v \Rightarrow[X]_{\mathrm{w}}=[X]_{\mathrm{v}}$.

Suppose $w^{*} \subseteq v$. Then $(X=Y) \in w<=(X=Y) \in v$, by ii). 
Thus $Y \in[X]_{\mathrm{w}}<=>\in[X]_{\mathrm{v}}$, and so $[X]_{\mathrm{w}}=[X]_{\mathrm{v}}$, by the definition of $[X]_{\mathrm{w}}$.

Suppose now $w^{*} \subseteq v$. Then $B_{\mathrm{w}}=B_{\mathrm{v}}$, by iii).

Hence $\left(B_{\mathrm{w}},+_{\mathrm{w}}, 1_{\mathrm{w}}, \gamma_{\mathrm{w}}, \mathfrak{I}_{\mathrm{w}}\right)=\left(B_{\mathrm{v}},+_{\mathrm{v}}, 1_{\mathrm{v}}, \gamma_{\mathrm{v}}, \mathfrak{I}_{\mathrm{v}}\right)$, by the definition of $+_{\mathrm{w}}, 1_{\mathrm{w}}, \gamma_{\mathrm{w}}$ and $\mathfrak{I}_{\mathrm{w}}$.

Truth Lemma: $M, w \mid=\varphi<=>\varphi w$.

The proof is by induction. The new case concerns identities.

$M, w \mid=X=Y<=>(X=Y) \in w$.

$M, w \mid=X=Y<=>\mathfrak{I}_{\mathrm{w}}(X)=\mathfrak{I}_{\mathrm{w}}(Y)<=>[X]_{\mathrm{w}}=[Y]_{\mathrm{w}}<=>(X=Y) \in w$, by fact 1.

In conclusion, $M$ is a model for $\mathbf{E}^{=}$satisfying $T C$ and $U C$ (see lemma 2.5.6 and lemma 2.5.7).

\section{The quantified systems}

In this section we show how it is possible to interpret two systems of quantified logic of essence by using Fitting semantics ${ }^{10}$. Let $\mathbf{L}$ be one of the systems of propositional logic of essence introduced before. The system $\mathbf{Q} \mathbf{L}$ of quantified logic of essence corresponding to $\mathbf{L}$ is defined as follows. The alphabet of the language, $L(\mathbf{Q L})$, includes the alphabet of the language $L(\mathbf{L})$ and contains, in addition, the following signs.

- the universal quantifier: $\forall$.

- the predicate of existence: $\boldsymbol{E}$ !

- a countable set $I V$ of individual variables $\left(x, x^{\prime}, x^{\prime \prime}, \ldots\right)$.

- for every $n$, a countable set of $n$-ary predicates $\left(P^{\mathrm{n}}, P^{\mathrm{n}}, P^{\mathrm{n},}, \ldots\right)$.

Remark: we will use " $\forall$ " to denote the universal quantifier both on the theoretical level and on the metatheoretical level. It will be always clear from the context which quantifier is denoted.

We have two kinds of terms: terms for delimiters and terms for individuals. The definitions of term and formula are expanded as follows.

Rules for terms of $L(\mathbf{Q L})$.

i) $|x|$ is a term, if $x$ is an individual variable;

ii) $|P|$ is a term, if $P$ is a predicate.

Rules for formulas of $L(\mathbf{Q L})$.

i) $P^{\mathrm{n}}(\boldsymbol{x})$ is a formula, if $\boldsymbol{x}$ is a sequence of $n$ individual variables;

ii) $\boldsymbol{E} !(x)$ is a formula, if $x$ is an individual variable;

iii) $\forall x \varphi$ is a formula, if $\varphi$ is a formula.

\footnotetext{
${ }^{10}$ See [8], Part III, for a detailed introduction to quantified modal logic.
} 
$\varphi[x:=y]$ denotes the result of the substitution of the individual variable $y$ for $x$ in $\varphi$.

Definition 4.1: ontological reference of $\varphi$.

Let $\varphi$ be a formula. Then, the ontological reference of $\varphi$ is defined as follows:

$$
\operatorname{Ref}(\varphi):=d_{1} \bullet \ldots \bullet d_{\mathrm{j}} \bullet\left|x_{1}\right| \cdot \ldots \cdot\left|x_{\mathrm{i}}\right| \bullet\left|P_{1}\right| \cdot \ldots \cdot\left|P_{\mathrm{h}}\right|, \text { where }
$$

i) $d_{1}, \ldots, d_{\mathrm{j}}$ are the variables for delimiters occurring in $\varphi$.

ii) $x_{1}, \ldots, x_{\mathrm{i}}$ are the variables occurring free in $\varphi$.

iii) $P_{1}, \ldots, P_{\mathrm{h}}$ are the predicates occurring in $\varphi$.

Intuitively, $\operatorname{Ref}(\varphi)$ is the plurality including all the entities $\varphi$ refers to.

QL is the extension of $\mathbf{L}$ determined by adding the following axioms and rules.

Group III: classical axioms and rules.

$\forall \mathbf{E}: \forall x \boldsymbol{E} !(x)$.

$\forall 1: \forall x \varphi \rightarrow \varphi[x:=y], y$ free for $x$ in $\varphi$.

$\forall \mathbf{2}:\left|-\forall x\left(\varphi \rightarrow \varphi^{\prime}\right)=>\right|-\varphi \rightarrow \forall x \varphi^{\prime}, x$ not free in $\varphi$.

Group IV: modal quantified axioms and rules.

\begin{tabular}{|l|l|}
\hline Axioms on delimiters: & Axioms on modalities: \\
\hline D9: $[|\varphi|] \varphi ’ \leftrightarrow[\operatorname{Ref}(\varphi)] \varphi$, & $\forall \mathbf{M : ~} \forall x[X] \varphi \rightarrow[X] \forall x \varphi$. \\
\hline
\end{tabular}

Note that D9 is a generalization of axioms D5-D8.

\subsection{Theorems}

QT1: $\mid-[X] \forall x \varphi \rightarrow \forall x[X] \varphi$.

$\mid-\forall x \varphi \rightarrow \varphi[x:=y], y$ free in $\varphi$, by pred. logic.

$\mid-[|\forall x \varphi \rightarrow \varphi[x:=y]|](\forall x \varphi \rightarrow \varphi[x:=y])$, by $\mathbf{R M}$.

$\mid-[|\forall x \varphi|](\forall x \varphi \rightarrow \varphi[x:=y])$, by D5, D6, D9.

$\mid-[X] \forall x \varphi \rightarrow[X \bullet|\forall x \varphi|] \varphi[x:=y]$, by T4.

$-[X] \forall x \varphi \rightarrow[X] \varphi[x:=y]$, by $\mathbf{T 5}$.

$-[X] \forall x \varphi \rightarrow \forall x[X] \varphi[x:=y]$, by pred. logic. 


\subsection{Semantics}

Definition 4.3.1: constituency structure / $\gamma$-structure.

See definitions 2.3.1 and 3.3.1.

Definition 4.3.2: constituency model / $\gamma$-model.

See definitions 2.3 .2 and 3.3.2. The only difference concerns $\mathfrak{I} 3$ :

$\mathfrak{\Im} 3: \mathfrak{\Im}(|\varphi|)=\mathfrak{\Im}(\operatorname{Ref}(\varphi))$

Definition 4.3.3: frame for $L(\mathbf{Q L})$.

A frame for $L(\mathbf{Q} \mathbf{L})$ is a tuple $(W, R, \mathbf{B}, E, I)$, where $(W, R, \mathbf{B}, E)$ is a frame for $L(\mathbf{L})$ and $I$ is a nonempty set, i.e. the domain of individuals.

Definition 4.3.4: model for $L(\mathbf{Q L})$.

A model for $L(\mathbf{Q} \mathbf{L})$ is a tuple $M=(W, R, \mathrm{~B}, E, I, V)$, where $V$ is an assignment such that:

i) $V(w, x) \in I$, for every $w \in W$ and individual variable $x$;

ii) $V(w, x)=V(v, x)$ for every $w, v \in W$ and individual variable $x$;

iii) $V\left(w, P^{\mathrm{n}}\right) \subseteq D^{\mathrm{n}}$, for every world $w \in W$ and $n$-ary predicate $P^{\mathrm{n}}$.

The $[x: i]$-variant of $V$ is the assignment $V[x: i]$ defined as follows:

i) $V[x: i](y)=i$, if $x=y$;

ii) $V[x: i](y)=V(y)$, otherwise.

If $M=(W, R, \mathrm{~B}, E, I, V)$, then $M[x: i]$ is the model $(W, R, \mathrm{~B}, E, I, V[x: i])$.

Definition 4.2.5: truth (in a model at a world).

In addition to the propositional conditions for $\mathbf{L}$, we have

$M, w \mid=P^{\mathrm{n}}\left(x_{1}, \ldots, x_{\mathrm{n}}\right)<=>\left(V\left(w, x_{1}\right), \ldots, V\left(w, x_{\mathrm{n}}\right)\right) \in V\left(w, P^{\mathrm{n}}\right)$.

$M, w \mid=\boldsymbol{E} !(x)<=>V(w, x) \in I$, i.e. $M, w \mid=\boldsymbol{E} !(x)$.

$M, w \mid=\forall x \varphi<=>\forall i \in I(M[x: i], w \mid=\varphi)$.

Definition 4.2.6: saturated model.

A model for $L(\mathbf{Q L})$ is said to be saturated when $E$ satisfies the following constraint:

$\forall x \varphi \in E(w, b)<=>\varphi[x:=y] \in E(w, b)$, for every individual variable $y$.

\subsection{Soundness}

Soundness is proved as usual. 
Theorem 4.3.1: suppose $\mathbf{L}$ is sound with respect to the class of all models for $L(\mathbf{L})$ satisfying $T C$ and $U C$. Then the corresponding quantified system $\mathbf{Q L}$ is sound with respect to the class of all saturated models for $L(\mathbf{Q L})$ satisfying $T C$ and $U C$.

We check M $\mathbf{M}: \|-\forall x[X] \varphi \rightarrow[X] \forall x \varphi$.

Suppose $M, w \mid=\forall x[X] \varphi$.

Then $\forall i \in I(M[x: i], w \mid=[X] \varphi)$.

$\forall i \in I(\forall v \in W(R(w, v)=>M[x: i], v \mid=\varphi)$.

$\forall v \in W(R(w, v)=>\forall i \in I(M[x: i], v \mid=\varphi)$.

$\forall v \in W(R(w, v)=>M, v \mid=\forall x \varphi)$.

By induction hypothesis, $M, w \mid=[X] \varphi[x:=y])$ for every $y$.

Hence $\varphi[x:=y] \in E(w, \mathfrak{\Im}(X))$, for every $y$.

Therefore $\forall x \varphi \in E(v, \Im(X))$, since $M$ is saturated, and so $M, w \mid=[X] \forall x \varphi$.

\subsection{Completeness}

Completeness is proved by a canonical model construction.

Say that a set $\Delta$ of formulas is complete in $L(\mathbf{Q L})$ if and only if

1) $\Delta$ is QL-closed;

2) $\Delta$ is $\mathbf{Q L}$-consistent;

3) for each $\varphi \in L(\mathbf{Q L}), \varphi \in \Delta$ or $\neg \varphi \in \Delta$.

Say that a set $\Delta$ of formulas is $\forall$-complete in $L(\mathbf{Q L})$ if and only if, for each $\varphi \in L(\mathbf{Q L})$ and individual variable $x$, there exists an individual variable $y$ such that $(\varphi[x:=y] \rightarrow \forall x \varphi) \in \Delta$. It is plain that every set that includes a $\forall$-complete set is $\forall$-complete in $L(\mathbf{Q L})$. The following lemmas are well-known (see [8], ch. 14, for details).

Lemma 4.4.1: every $\mathbf{Q L}$-consistent set can be extended to a complete set in $L(\mathbf{Q L})$.

Lemma 4.4.2: every $\mathbf{Q L}$-consistent set can be extended to a $\forall$-complete set in $L^{*}(\mathbf{Q} \mathbf{L})$.

In the second lemma, $L^{*}(\mathbf{Q L})$ is an extension of $L(\mathbf{Q L})$ containing a countable set of new individual constants. It follows from the previous lemmas that, for every QL-consistent set $\Delta$, there exists a set $\Delta^{*}$, including $\Delta$, that is both $\forall$-complete and complete in $L^{*}(\mathbf{Q L})$. This is true with respect to any system which contains standard axioms for first order quantified logic.

Theorem 4.4.1: suppose $\mathbf{L}$ is complete with respect to the class of all models for $L(\mathbf{L})$ satisfying $T C$ and $U C$. Then, the corresponding quantified system $\mathbf{Q L}$ is complete with respect to the class of all saturated models for $L(\mathbf{Q} \mathbf{L})$ satisfying $T C$ and $U C$.

Definition 4.4.1: canonical constituency model. 
The canonical constituency model $\mathrm{B}_{\mathbf{Q L}}=\left(B_{\mathbf{Q L}},{ }_{\mathbf{Q L}}, l_{\mathbf{Q L}}, \mathfrak{\Im}_{\mathbf{Q L}}\right)$ is defined as follows.

$B_{\mathbf{Q L}}=\left\{[X]_{\mathrm{QL}} \mid X\right.$ a delimiter $\}$, where $[X]_{\mathrm{QL}}=[X]_{\mathrm{L}}$;

${ }^{+}{ }_{\mathbf{Q L}}$ is such that $[X]_{\mathbf{Q L}}{ }_{\mathbf{Q L}}[Y]_{\mathbf{Q L}}=[X \cdot Y]_{\mathbf{Q L}}$ and $l_{\mathbf{Q L}}=[V]_{\mathbf{Q L}}$;

$\mathfrak{\Im}_{\mathbf{Q L}}$ is such that $\mathfrak{I}_{\mathbf{Q L}}(X)=[X]_{\mathbf{Q L}}$.

Conditions $\mathfrak{\Im} 1-\mathfrak{\Im} 3$ / $\mathfrak{\Im} 1-\mathfrak{I} 4$ on $\mathfrak{\Im}_{\mathbf{Q L}}$ are satisfied by $\mathbf{D 9}$.

Definition 4.4.2: canonical model for $L^{*}(\mathbf{Q L})$.

The canonical model for $L(\mathbf{Q L})$ is the tuple $M_{\mathbf{Q L}}=\left(W_{\mathbf{Q L}}, R_{\mathbf{Q L}}, \mathrm{B}_{\mathbf{Q L}}, E_{\mathbf{Q L}}, I_{\mathbf{Q L}}, V_{\mathbf{Q L}}\right)$, where:

$W_{\mathrm{QL}}$ is the set of $\forall$-complete sets.

$R_{\mathbf{Q L}}, \mathrm{B}_{\mathbf{Q L}}, E_{\mathbf{Q L}}$ are defined as in section 2 .

$I_{\mathbf{Q L}}$ is the set of all individual constants of $L^{*}(\mathbf{Q E})$.

$V_{\mathbf{Q L}}$ is such that:

1) $V_{\mathbf{Q L}}(w, x)=x$, for each individual variable of $L^{*}(\mathbf{Q E})$

2) $V_{\mathbf{Q L}}\left(w, P^{\mathrm{n}}\right)=\left\{\boldsymbol{x} \mid \boldsymbol{x}\right.$ is a sequence of $n$ individual variables and $\left.P^{\mathrm{n}}(\boldsymbol{x}) \in w\right\}$

In order to prove completeness we have to show that $M_{\mathbf{Q L}}$ is a saturated model for $L^{*}(\mathbf{Q L})$ satisfying $T C$ and $U C$ and is such that the truth lemma is provable with respect to it. Much of the proof is a direct carry-over of the corresponding proof for $\mathbf{L}$ and for standard systems of quantified modal logic. The only new part that concerns us is to show that $M_{\mathbf{Q L}}$ is a saturated model, which follows from this lemma.

Lemma 4.4.3: $\forall x \varphi \in E\left(w,[X]_{\mathrm{QL}}\right)<=>\varphi[x:=y] \in E\left(w,[X]_{\mathrm{QL}}\right)$.

Hence, we have to show that $\forall x \varphi \in w / X<\Rightarrow \varphi[x:=y] \in w / X$, for every $y$.

Suppose $\forall x \varphi \in w / X$. Then, $w / X \mid-\forall x \varphi$, and so $w / X \mid-\varphi[x:=y]$, for every $y$.

Thus $w \mid-[X] \varphi[x:=y]$, for every $y$, so that $[X] \varphi[x:=y] \in w$ and $\varphi[x:=y] \in w / X$, for every $y$.

Suppose $\forall x \varphi \notin w / X$. Then, $[X] \forall x \varphi \notin w$, and so $\forall x[X] \varphi \notin w$, by $\forall \mathbf{M}$.

Thus $[X] \varphi[x:=y] \notin w$ for some $y$, since $w$ is $\forall$-complete, so that $\varphi[x:=y] \notin w / X$ for some $y$.

\section{Conclusion}

In this paper a Fitting semantics was employed and developed in order to provide an intuitive framework within which to treat a number of systems of logic of essence. What are the advantages and disadvantages of this semantics with respect to the one proposed in [2] and [5]?

Advantages. There are two main advantages provided by the proposed semantics: 1) it gives a uniform semantic framework for interpreting different systems of logic of essence, both propositional and quantified; and 2) it builds a bridge between the logic of essence and the logic of evidence. In particular, the significance of point 2) is that we now have the possibility of exploiting the semantic resources of the logic of evidence both in order to show how the 
concepts of metaphysical necessity and ontological dependence can be defined on the basis of the concept of essence, and in order to produce new systems of logic of essence by making use of the systems of logic of evidence that are currently well-known.

Disadvantages. The highlighted flexibility comes at a cost. Indeed, while the frame for a logic of essence allows us to associate both formulas and terms for delimiters with appropriate ontological counterparts, namely sets of possible worlds and pluralities of basic entities, the notion of truth in virtue of the essence of certain entities is left unanalysed. In particular, in our semantics there is neither an ontological relation of truth in virtue of nor an ontological relation that can be used to define the notion of essential truth. As a consequence, the Fitting part of the semantics allows us to state, in a precise way, our intuitions about the logical structure of an operator like $[X]$, but it is of no help if our aim is to get a deeper understanding of this operator. Hence the generality of the current approach, which is independent of the particular ontological construction of the notion of essence for which we opt, contrasts with the task of explaining why a certain proposition is true in virtue of the essence of certain entities. By contrast, in [2] and in [6] the notion of essential truth is analysed with reference to specific ontological structures, so that we are in a position to explain why a number of principles concerning $[X]$ are taken as valid.

A future line of research would then be to adapt the present semantic framework so as to be able to define the notion of essential truth directly in terms of ontological dependence. In addition, it would be of interest to investigate whether such a framework has to be changed if delimiters are identified, following Fine, with sets of individuals.

\section{References}

[1] S. Artemov, Logic of Justification, Review of Symbolic Logic, 1: 477-513, 2008.

[2] F. Correia, A Propositional Logic of Essence, Journal of Philosophical Logic, 29: 295-313, 2000

[3] K. Fine, Essence and Modality. Philosophical Perspectives, 8: 1-16, 1994.

[4] K. Fine, Ontological Dependence, Proc. of the Aristotelian Society, XCV: 269-290, 1995.

[5] K. Fine, The Logic of Essence, Journal of Philosophical Logic, 24: 241-273, 1995.

[6] K. Fine, Semantics for the Logic of Essence, Journal of Philosophical Logic, 29: 543-584, 2000

[7] M. Fitting, The Logic of Proofs, Semantically, Annals of Pure and Applied Logic, 132: 1-25, 2005.

[8] G.E. Hughes \& M.J. Cresswell, A New Introduction to Modal Logic, Routledge, London 1996. 